\title{
On the Numerical Performance of a New Conjugate Gradient Parameter for Solving Unconstrained Optimization Problems
}

\author{
Aliyu Usman Moyi \\ Department of Mathematical Sciences \\ Faculty of Science \\ Federal University Gusau, Zamfara State, Nigeria
}

\author{
Onwuka Blessing \\ Department of Mathematical Sciences \\ Faculty of Science \\ Federal University Gusau, Zamfara State, Nigeria
}

\begin{abstract}
Nonlinear Conjugate gradient methods (CG) are widely used for solving unconstrained optimization problems. Their wide application in many Fields such as Engineering, Applied Sciences and Economics is due to their low memory requirements and global convergence properties. Numerous studies and modifications directed towards improving the efficiency of these methods have been conducted. In this paper, a new conjugate gradient parameter $\beta_{k}$ that possess convergence properties is presented. We also present preliminary numerical results to show the efficiency of the proposed method.
\end{abstract}

\section{Keywords}

Unconstrained Optimization, Conjugate Gradient Method, Conjugate Gradient Coefficient, Global Convergence.

\section{INTRODUCTION}

In this paper, we deal with the conjugate gradient (CG) methods for the numerical solution of the unconstrained optimization problem

$$
\min f(x), x \in R^{n}
$$

where $f: R^{n} \rightarrow R$ is assumed to be atleast twice continuously differentiable function and $n$ is the dimension of $x$, which is assumed to be large. The iterates of the conjugate gradient methods are obtained by

$$
x_{k+1}=x_{k}+\alpha_{k} d_{k},
$$

where $x_{k}$ is the current iterate point, $d_{k}$ is the search direction and $\alpha_{k}>0$ is the step length. The success of any conjugate gradient method depends on the effective choices of both the search direction and the step length. Two strategies for calculating the step length is the exact line search and inexact line search method. The ideal choice would be the exact line search which is defined by

$$
f\left(x_{k}+\alpha_{k} d_{k}\right)=\min _{\alpha \in R} f\left(x_{k}+\alpha_{k} d_{k}\right)
$$

but in general it is computationally expensive to obtain since it requires too many evaluations of the objective function $f$ and its gradient $g$. The other alternative choice is the inexact line search strategies such as the Armijo line search [7], Wolfe condition [] and Goldstein condition [2]. The conjugate gradient methods also define the search direction $d_{k}$ by

$$
d_{k+1}=-g_{k+1}+\beta_{k} d_{k}, \quad d_{0}=-g_{0}
$$

for $k \geq 1$, where the parameter $\beta_{k} \in R$ is a scalar known as conjugate gradient coefficient.

In the literature, several choices for $\beta_{k}$ have been proposed which give rise to distinct conjugate gradient methods. The most well-known conjugate gradient methods are the Hestenes-Steifel (HS)[10], Fletcher-Reeves(FR)[11], Polak-Ribeire (PR) [3], Liu-Storey (LS),[13] Dai-Yuan (DY)[12] and Gilbert-Nocedal $(\mathrm{PR}+)[9]$ in which the update parameter of these methods is respectively specified as follows:

$\beta_{k}^{H S}=\frac{y_{k}^{T} g_{k+1}}{y_{k}^{T} d_{k}}, \quad \beta_{k}^{F R}=\frac{g_{k+1}^{T} g_{k+1}}{g_{k}^{T} g_{k}}, \quad \beta_{k}^{P R}=\frac{y_{k}^{T} g_{k+1}}{g_{k}^{T} g_{k}}$,

$\beta_{k}^{L S}=\frac{-y_{k}^{T} g_{k+1}}{g_{k}^{T} d_{k}}, \quad \beta_{k}^{D Y}=\frac{g_{k+1}^{T} g_{k+1}}{y_{k}^{T} d_{k}}, \quad \beta_{k}^{P R+}=$ $\max \left\{\frac{y_{k}^{T} g_{k+1}}{g_{k}^{T} g_{k}}, 0\right\}$,

where $s_{k}=x_{k+1}-x_{k}$ and $y_{k}=g_{k+1}-g_{k}$. Note that these formulae for $\beta_{k}$ are equivalent to each other if the objective function is a strictly convex quadratic function and $\alpha_{k}$ is chosen through an exact line search. However, for general non-quadratic functions or under the inexact line search their behavior is quite different [6].

The rest of this paper is organized as follows. In the next section, we simply recall the conjugate gradient direction for solving unconstrained optimization problem and construct our new conjugate parameter subsequently. In section 3 , we present the convergence of the proposed algorithm. Then we present some preliminary results of the new approach on some standard test problems and finally, we conclude the paper in section 5 .

\section{THE NEW CG PARAMETER}

We proposed our modification based on the search direction from Ibrahim et.al [5] given as

$$
d_{k+1}=-B_{k+1}^{-1} g_{k+1}+\lambda_{k+1} d_{k},
$$


where $B_{K+1}$ is the BFGS updating matrix and $\lambda_{k+1}=$ $\eta g_{k+1}^{T} g_{k+1} / g_{k+1}^{T} d_{k}$ with $\eta \in(0.1]$ is chosen to ensure conjugacy. from (4) and (5) we have,

$$
-g_{k+1}+\beta_{k+1} d_{k}=-B_{k+1}^{-1} g_{k+1}+\lambda_{k+1} d_{k},
$$

multiply (6) by $s_{k+1}^{T} B_{k+1}$

$$
\begin{aligned}
& -s_{k+1}^{T} B_{k+1} g_{k+1}+\beta_{k+1} d_{k} s_{k+1}^{T} B_{k+1}=-g_{k+1}^{T} s_{k+1}+\lambda_{k+1} \\
& \text { But, } B_{k+1} s_{k+1}=y_{k+1} \text {, then, } \\
& -g_{k+1}^{T} y_{k+1}+\beta_{k+1} d_{k}^{T} y_{k+1}=-g_{k+1}^{T} s_{k+1}+\lambda_{k+1} d_{k}^{T} y_{k+1} \\
& \beta_{k+1} d_{k}^{T} y_{k+1}=-g_{k+1}^{T} s_{k+1}+\lambda_{k+1} d_{k}^{T} y_{k+1}+g_{k+1}^{T} y_{k+1} \\
& \beta_{k+1}=\frac{-g_{k+1}^{T} s_{k+1}+\lambda_{k+1} d_{k}^{T} y_{k+1}+g_{k+1}^{T} y_{k+1}}{d_{k}^{T} y_{k+1}}
\end{aligned}
$$

Thus,

$$
\beta_{k+1}=\frac{\left(\lambda_{k+1} d_{k}+g_{k+1}\right) y_{k+1}^{T}-g_{k+1}^{T} s_{k+1}}{d_{k}^{T} y_{k+1}}
$$

Similarly, $d_{k}=s_{k}$

$$
\beta_{k+1}^{N E W}=\frac{\left(\lambda_{k+1} s_{k}+g_{k+1}\right) y_{k+1}^{T}-g_{k+1}^{T} s_{k+1}}{s_{k}^{T} y_{k+1}} .
$$

\section{New Algorithm}

We now present the basic steps of the algorithm for solving unconstrained optimization problems as;

Step 1 : Given an initial point $x_{0} \in R^{n}$, set $d_{0}=-g_{0}$ and $k=0$

Step 2 : Test a criterion for stopping the iterations. If the test is satisfied, then stop; otherwise continue with step 3

Step 3 : Compute the search direction $d_{k}$ by (4), with $\beta_{k+1}$ defined in (12)

Step 4 : Find an acceptable steplength $\alpha_{k}$, by using the following line search procedure. Given the constants $\eta \in(0,1)$ and $\tau, \tau^{\prime}$ with $0<\tau<\tau^{\prime}<1$

(i) $\operatorname{Set} \alpha=1$

(ii) Test the relation

$$
f\left(x_{k}+\alpha d_{k}\right) \leq f\left(x_{k}\right)+\eta \alpha g_{k}^{T} d_{k},
$$

(iii) If 13 is not satisfied, choose a new $\alpha$ in $\left[\tau \alpha, \tau^{\prime} \alpha\right]$ and go to (ii). If (13) is satisfied, set $\alpha_{k}=\alpha$ and $x_{k+1}=x_{k}+\alpha_{k} d_{k}$

Step $5:$ Set $k:=k+1$, and go to step 2

\section{NUMERICAL RESULTS AND DISCUSSIONS}

In this section, we present some numerical results from an implementation of our new conjugate gradient algorithm for solving unconstrained optimization problems, we evaluate the performance of oue new conjugate gradient parameter with that of

(1) Polak-Ribeire (PR)[3] ,

(2) Hestenes-Steifel (HS)[10] HS:
All the experiments are implemented on a PC using MATLAB version 7.13.0.564 (R 2011b), with double precision arithmetic. For each test function, we perform 40 numerical experiments with variable dimensions $50 \leq n \leq 1000$. As regards the stopping criteria used in our experiments, in all the algorithms, convergence is assumed if $\left\|g_{k}\right\| \leq \varepsilon$ where $\varepsilon=10^{-4}$. We forced the algorithm to stop whenever the number of iterations exceeds 2000, and the symbol "." is used to represent the failure. Test functions are the standard unconstrained optimization problems obtained from [1] $B_{k+\text { as }}$ presentesd in the Table below.

(7) Table 1 gives the performance of all the algorithms, where a total of 20 runs are performed. Based on the results, $\beta_{k}^{N e w}$ solves $100 \%$ of the test problems while PR, HS, can only solve $80 \%$ and $90 \%$ of the test problems respectively. The performance of $\beta_{k}^{\text {New }}$ over PR, is that New $\beta_{k}^{\text {New }}$ needs $48 \%$ and $78 \%$ less in terms of the number of iterations than PR and HS. Overall, we believe that given the ratio of iteration counts for all the methods, we can conclude that our algorithm is promising even when less accurate line search strategy is employed.

\section{CONCLUSION}

Numerous studies on CG method have resulted in different conjugate gradient parameters. Although, these variety of methods have been shown to perform much better than the classical methods they have been reported to be complex and difficult to implement in practice. Therefore, identifying these shortcomings and rectifying them as modified methods for efficient performance is worthwhile. Thus, in this paper we have presented a new conjugate gradient parameter for solving unconstrained optimization problems. The numerical results for a small dimension of the test problems show that the new parameter is efficient and robust.

\section{REFERENCES}

[1] Andrei, N.: An unconstrained optimization test functions collection, Adv. Model. Optim. 10(1), 147-161 (2008)

[2] Goldstein,A. A.On Steepest Descent SIAM J. Control. 3(1965) 147-151.

[3] Polak, E.,Ribiere, Note sur la convergence de directions conjugees,Rev. Francaise Inform. Recherche Opetationelle 3 (1969) 36-43.

[4] Byrd, R. H., Nocedal, J.: A tool for the analysis of quasi-Newton methods with pplication to unconstrained minimization. SIAM J. Numer. Anal. 26(3), 727-739 (1989)

[5] Ibrahim, M. A , Mamat M. and Leong W, J.”:BFGS Method: A New Search Direction. Sains Malaysiana 42(10) 2014, 1593-1599

[6] Dai, Y. H.: On the nonmonotone line search. J. Optim. Theory Appl. 112(2), 315-330 (2002)

[7] Armijo, L. Minimization of Functions having Lipschitz Continuous Partial Derivatives, Pacific J. Math 16(1966)1-3

[8] Dolan, E.D., More, J.J.: Benchmarking optimization software with performance profiles. Math. program. 91 (2), 201-213 (2002) [9] Gilbert, J.C., Nocedal, J.: Global convergence properties of conjugate gradient methods for optimization. SIAM J. Optim. 2 (1), 21-42 (1992)

[10] Hestenes. M.R., Steifel, E. Method of Conjugate Gradient for Solving Linear Equations, J. Res. Nat. Bur. Stand.49 (1952) 409436. 
Table 1. Numerical Results of PR, HS, and New

\begin{tabular}{|c|c|c|c|c|}
\hline \multicolumn{5}{|c|}{$\beta_{k}$} \\
\hline \multirow[t]{2}{*}{ Test Fuction } & Dim. & PR & HS & $\beta_{k+1}^{N e w}$ \\
\hline & & NI & NI & NI \\
\hline Extended & 50 & - & 43 & 33 \\
\hline Freudeinstein & 100 & - & 43 & 33 \\
\hline \multirow[t]{2}{*}{ and Roth } & 500 & - & 47 & 34 \\
\hline & 1000 & - & 47 & 36 \\
\hline Extended & 50 & 115 & 47 & 39 \\
\hline \multirow[t]{3}{*}{ Beal } & 100 & 117 & 47 & 39 \\
\hline & 500 & 121 & 50 & 39 \\
\hline & 1000 & 123 & 49 & 42 \\
\hline Extended & 50 & 40 & 76 & 20 \\
\hline Block & 100 & 35 & 69 & 21 \\
\hline \multirow[t]{2}{*}{ Diagonal 1} & 500 & 38 & 70 & 19 \\
\hline & 1000 & 40 & 70 & 19 \\
\hline \multirow[t]{4}{*}{ Raydan 2} & 50 & - & 276 & 18 \\
\hline & 100 & - & 352 & 18 \\
\hline & 500 & - & 606 & 20 \\
\hline & 1000 & - & 776 & 25 \\
\hline Generalized & 50 & 31 & 104 & 20 \\
\hline \multirow[t]{3}{*}{ Tridiagonal 1} & 100 & 33 & 105 & 21 \\
\hline & 500 & 35 & 105 & 22 \\
\hline & 1000 & 35 & 105 & 22 \\
\hline \multirow[t]{4}{*}{ Diagonal 4} & 50 & 106 & 53 & 24 \\
\hline & 100 & 129 & 55 & 24 \\
\hline & 500 & 113 & 57 & 24 \\
\hline & 1000 & 127 & 59 & 25 \\
\hline Extended & 50 & 29 & 186 & 17 \\
\hline \multirow[t]{3}{*}{ Himelblau } & 100 & 29 & 192 & 18 \\
\hline & 500 & 31 & 205 & 19 \\
\hline & 1000 & 31 & 211 & 19 \\
\hline Extended & 50 & 33 & 24 & 10 \\
\hline \multirow[t]{3}{*}{ Psc } & 100 & 33 & 24 & 10 \\
\hline & 500 & 35 & 24 & 10 \\
\hline & 1000 & 37 & 24 & 11 \\
\hline Extended & 50 & 51 & - & 35 \\
\hline \multirow[t]{3}{*}{ TCliff } & 100 & 51 & - & 35 \\
\hline & 500 & 100 & - & 35 \\
\hline & 1000 & 75 & - & 35 \\
\hline Extended & 50 & 31 & 68 & 20 \\
\hline Three Exponential & 100 & 33 & 70 & 21 \\
\hline \multirow[t]{2}{*}{ Terms } & 500 & 35 & 76 & 22 \\
\hline & 1000 & 35 & 80 & 22 \\
\hline
\end{tabular}

[11] Fletcher, R. Reeves, C. Function Minimization by Conjugare Gradients, Comput. J. 7 (1964) 149-154.

[12] Dia, Y. H., Yuan, Y. Nonlinear Conjugate Gradient Method, Shanghai Scientific and Technical Publishers, Beijing , 1998

[13] Liu, Y., Storey, C. Efficient Generalized Conjugate Gradient Algorithms Part 1:Theory,J.Comput.Appl.Math. 69(1992) 129137.

[14] Panier, E.R., Tits, A.L.: Avoiding the maratos effect by means of a nonmonotone line search I. General constrained problems. SIAM J. Numer. Anal. 28(4), 1183-1195 (1991)

[15] Perry, J.M.: A class of conjugate gradient algorithms with a two step variable metric memory. Center for Mathematical Studies in Economies and Management Science. Evanston Illiois: Northwestern University Press. 1977
[16] Sun, W., Han, J., Sun, J.: Global convergence of nonmonotone descent methods for unconstrained optimization problems. J. Comput. Appl. Math. 146(1), 89-98 (2002)

[17] Shi, Z.J., Wang, S., Xu, Z.: The convergence of conjugate gradient method with nonmonotone line search. Appl. Math. Comput. 217(5), 1921-1932 (2010)

[18] Yuan, G., Wei, Z.: Non monotone backtracking inexact BFGS method for regression analysis. Comm. Stat. Theory Meth. 42(2), 214-238 (2013) 